\title{
Scolia
}

Revue de linguistique

30 | 2016

Des connecteurs argumentatifs aux opérateurs discursifs

\section{Opérateurs discursifs en revanche et par contre}

\section{Patrick Haillet}

\section{(2) OpenEdition}

Journals

Édition électronique

URL : http://journals.openedition.org/scolia/511

DOI : 10.4000/scolia. 511

ISSN : 2677-4224

Éditeur

Presses universitaires de Strasbourg

\section{Édition imprimée}

Date de publication : 19 juin 2016

Pagination : 123-137

ISBN : 978-2-86820-944-3

ISSN : 1253-9708

Référence électronique

Patrick Haillet, "Opérateurs discursifs en revanche et par contre », Scolia [En ligne], 30 | 2016, mis en ligne le 09 avril 2019, consulté le 22 mai 2019. URL : http://journals.openedition.org/scolia/511 ; DOI : 10.4000/scolia.511 
SCOLIA 30/2016, p. 123-138.

\title{
Opérateurs discursifs en revanche et par contre
}

\author{
Patrick Haillet \\ Université de Cergy-Pontoise, \\ UMR 7187 LDI \\ hailletpatrick@yahoo.fr
}

\section{Introduction}

Cette contribution vise à rendre compte de certaines propriétés sémantiques des entités en revanche et par contre. Pour ce faire, on s'appuiera sur le postulat selon lequel le discours - ensemble plus ou moins complexe de séquences sonores ou écrites associées à du sens, présentant la double articulation et produites (ou susceptibles de se trouver produites) spontanément par un locuteur natif - a pour fonction fondamentale de représenter «ce dont on parle» d'une certaine manière, la tâche du linguiste étant de préciser la nature des représentations construites par le discours en cherchant à mettre au jour les caractéristiques jugées pertinentes des constructions observées.

Après avoir esquissé les grands principes de la démarche adoptée, on s'attachera à examiner les particularités des représentations discursives contenant les opérateurs en revanche et par contre, l'objectif poursuivi étant de faire apparaître leur invariant sémantique.

\section{Mise en relation de points de vue et opérateurs discursifs}

L'approche présentée dans ce qui suit part du principe que le discours constitue fondamentalement un agencement de points de 
vue sur ce dont on parle - un point de vue étant ou bien exprimé par un segment de discours, ou bien paraphrasable par un segment de discours. La démarche exposée dans cette étude s'inspire des travaux de Ducrot et Anscombre sur la polyphonie et sur «l'argumentation dans la langue» (Anscombre \& Ducrot, 1976, 1977, 1981, 1983; Ducrot, 1980, 1984); par convention, elle réserve l'appellation d'énoncé à tout segment de discours organisé autour d'un verbe conjugué.

Dans la perspective adoptée ici, produire un énoncé revient à mettre en place au moins un point de vue. Ainsi, par exemple, tant C'est un traitement efficace que C'est un traitement contraignant - interprétés par défaut - véhiculent chacun un point de vue unique sur l'objet discursif correspondant, alors que C'est un traitement efficace mais contraignant met en relation les points de vue "C'est un traitement efficace» et "C'est un traitement contraignant» en les représentant comme arguments favorisant respectivement deux types opposés de conclusions (p. ex. "Je ne regrette pas de l'avoir choisi», déductible du premier, et "Je regrette de l'avoir choisi», déductible du second). En outre, l'exemple C'est un traitement efficace mais contraignant ne se combine pas avec des suites telles que J'en suis très satisfait (type correspondant aux conclusions favorisées par "C'est un traitement efficace») alors qu'il admet très naturellement des enchaînements tels que Un patient sur deux l'abandonne spontanément au bout de quelques semaines (type correspondant aux conclusions favorisées par "C’est un traitement contraignant») - ce qui conduit à dire que le segment introduit par mais est représenté comme privilégié discursivement par le locuteur-auteur de l'énoncé et comme déterminant tant les enchaînements naturels que les enchaînements incongrus. On adoptera en outre la position de Nølke (2006a: 39), en précisant que ces deux types de "conclusions" - implicites - déductibles respectivement de ce qui précède et de ce qui suit l'opérateur mais constituent des listes ouvertes, la description linguistique du phénomène reposant sur la relation d'opposition entre ces deux «classes».

Les constructions discursives qui mettent en relation deux points de vue sont d'une grande diversité; il convient tout d'abord de préciser qu'elles ne reposent pas toutes sur l'emploi d'un «marqueur attitré». C'est ce que l'on constate, par exemple, à propos de la "stratégie concessive» (Haillet, 2004) à l'œuvre dans Ils seraient milliardaires, ça ne changerait rien - ou encore à propos de la «stratégie de l'ironie» 
illustrée par C'est intelligent de les habiller tout en noir alors que c'est la canicule (Haillet, 2014). Dans ce qui suit, on focalisera l'attention sur les agencements où la mise en relation de deux points de vue s'opère moyennant le recours à un opérateur discursif - en d'autres mots, à un segment de discours identifiable.

On notera, à propos de ce dernier type de construction discursive, que plusieurs cas se présentent sur le plan de la structure syntaxique des agencements interprétés comme mettant en relation deux points de vue. Dans Je suis content parce que je visais la deuxième place, l'opérateur parce que met en relation le point de vue correspondant au segment de discours constituant son cotexte gauche avec celui qui correspond au segment de discours constituant son cotexte droit. Dans Comme il était tôt, il n'y avait pas beaucoup de clients, l'opérateur comme met en relation les points de vue «Il était tôt » et «Il n'y avait pas beaucoup de clients", correspondant tous les deux à des segments de discours situés dans son cotexte droit. Dans J'étais sauvé; je le croyais du moins, l'opérateur du moins met en relation les points de vue «J'étais sauvé» et "Je le croyais», correspondant tous les deux à des segments de discours situés dans son cotexte gauche. Enfin, la procédure visant à déterminer quels sont les points de vue mis en relation par l'emploi d'un opérateur discursif peut s'avérer bien plus complexe là où un point de vue explicitement exprimé se trouve mis en rapport avec un point de vue implicite (cf. par exemple Bruxelles et al., 1976 à propos de l'opérateur mais; Nølke, 2002, 2006a à propos de l'opérateur donc; Haillet, 2010 à propos de l'opérateur au moins).

C'est dans cette optique que sera abordé ici le fonctionnement des entités en revanche et par contre. Si, pour des raisons « pédagogiques » qui semblent évidentes, les exemples cités dans de nombreux dictionnaires présentent le plus souvent la structure «A en revanche $\mathrm{B}^{1}$ » / $\mathrm{A}$ par contre $\mathrm{B}^{2} »$ (les points de vue $\mathrm{A}$ et $\mathrm{B}$ mis en relation correspondant respectivement au cotexte gauche et au cotexte droit de l'opérateur), il convient de préciser, d'entrée de jeu, que les occurrences des deux opérateurs sont fréquemment attestées dans des environnements où un

1 Par exemple, La moisson a été médiocre, en revanche, la récolte des fruits a été excellente (Dictionnaire du Français Contemporain, 1980).

2 Par exemple, C'est un garçon charmant; par contre, son frère a un caractère détestable (Dictionnaire du Français Contemporain, 1980). 
point de vue explicite se trouve - du fait de l'emploi de par contre ou de en revanche - mis en relation avec un point de vue implicite.

C'est ce qu'illustre l'exemple (1), produit par une caissière qui s'adresse ainsi à un client ayant mis sur le tapis roulant tous ses achats sauf un pack de six bouteilles de Volvic, resté dans le caddie (cas signalé et commenté dans Hamma \& Haillet, 2002: 107):

1) Par contre, la Volvic, il va falloir la sortir!

L'énoncé La Volvic, il va falloir la sortir! se trouve mis en relation avec une entité sémantique qui n'est pas exprimée par un segment de discours explicite. Le point de vue qui correspond à la séquence sonore La Volvic, il va falloir la sortir! est ici mis en contraste avec un point de vue implicite - paraphrasable, par exemple, par «Les articles de plus de $8 \mathrm{~kg}$ restent dans le chariot ${ }^{3} »$.

C'est un cas de figure à cet égard similaire qui se trouve illustré par l'extrait suivant comportant une occurrence du syntagme en revanche:

2) C'est à moi que tu dois faire confiance, Terradillos. À moi, la compatriote de tes aïeux. À moi, parce qu'Alejandro m'a raconté toute sa vie, la vraie, l'intime, la scabreuse. Tu sais sûrement que c'est sa grand-mère qui l'a élevé, une femme qui avait dî s'endurcir à force d'affronter la vie toute seule. Je la plains, la pauvre, parce que ça, en revanche, je connais. (Manguel, Tous les hommes sont menteurs, 2009)

Ici, le point de vue correspondant à «ça, je connais» est mis en contraste avec un point de vue implicite paraphrasable, par exemple, par «je ne connais pas tout» (ou encore "même s'il y a beaucoup de choses que je ne connais pas $^{4} »$, etc.).

Dans ce qui suit, les opérateurs en revanche et par contre seront par conséquent appréhendés sous un angle spécifique. L'hypothèse de base étant que leur emploi met fondamentalement en relation un point de vue explicite $\mathrm{B}$ avec un point de vue explicite ou implicite A,

3 Ou encore - cf. Hamma \& Haillet (2002:107) - par «Il n'est pas nécessaire de sortir du chariot les packs de Cristaline» (autre marque d'eau minérale), etc.; il s'agit, là encore, de listes ouvertes, le point de vue A étant - pour reprendre l'heureuse formule de Nølke (2006a: 41) - «inféré de la situation énonciative».

4 Là encore, on retrouve le phénomène illustré supra: le point de vue $\mathrm{A}$ - implicite est inféré de la situation énonciative, la séquence ça, en revanche, je connais étant interprétée en rapport avec les circonstances (Prieto, 1966) de sa production, c'est-àdire dans son environnement discursif (Haillet, 2007). 
on s'attachera à préciser quel «statut discursif» est conféré au point de vue explicite $\mathrm{B}$ par le recours à l'opérateur employé.

\section{Nature et fonction(s) des représentations «en revanche $B$ » et "par contre $\mathrm{B}$ »}

Par convention, on adoptera les schémas «en revanche $\mathrm{B}$ » et "par contre $\mathrm{B}$ " pour désigner les agencements discursifs mettant ainsi en relation le point de vue explicite B avec un point de vue (explicite ou implicite) A; pour ce qui est des configurations syntaxiques observées, et notamment de la place (cf. Hamma \& Haillet, 2002; Nølke, 2006b) qu'occupe l'opérateur par rapport au segment de discours correspondant au point de vue - explicite - $\mathrm{B}$, on constate trois cas de figure.

Ainsi, dans les exemples (3) et (4), l'opérateur introduit le segment de discours correspondant au point de vue B (respectivement, je suis content d'entendre des architectes dire vouloir innover en proposant des immeubles de caractère et je pense que je n'ai pas donné assez d'exemples):

3) Jesuisétudiant. Nous avions proposéque l'on aménage un campus qui réunisse les logements des étudiants. Cela nous aurait permis de faire la fête le soir sans déranger les voisins. Malheureusement, cette option n'a pas été choisie. En revanche, je suis content d'entendre des architectes dire vouloir innover en proposant des immeubles de caractère. (http://www.cergypontoise.fr/jcms/ p2_70829/fr/paroles-dexperts, 10/8/2015)

4) J'ai réussi à dire des choses basiques sur la conscience mais j'ai placé peu de citations. La raison est simple: je n'en connais qu'une! C'est "Je pense, donc je suis» de Descartes. Ce qui est bien, c'est qu'elle passe avec presque tous les sujets. Du coup, j'ai réussi à la placer sans problème. Par contre, je pense que je n'ai pas donné assez d'exemples... J'espère que ça ne va pas me pénaliser. (20 Minutes, 17/06/2015)

Dans (5) et (6), le segment de discours correspondant au point de vue $\mathrm{B}$ - respectivement, Ce travail n'est à faire qu'une fois et J'ai peu dormi - constitue le cotexte gauche de l'opérateur:

5) Contrairement à ce que j'aurais pu penser, l'intégration des ressources dans la plate-forme (le LMS) a pris un temps 
considérable (près de 80 heures). Ne vous laissez pas surprendre en pensant que vous pourrez tout intégrer à la dernière minute. Ce travail n'est à faire qu'une fois en revanche, et heureusement. Pour la deuxième et la troisième édition, on fait un "Export» du cours, puis un "Import» du fichier généré dans la nouvelle édition, et le tour est joué. (http://blog.educpros.fr/matthieucisel/2015/01/28/mooc-creer-des-activites-pertinentes-et-peucouteuses/, 10/8/2015)

6) Oui merci un week-end sympa... sport, victoire en hockey dans une ambiance énorme, et dimanche petit neveu adorable... J'ai peu dormi par contre et là c'est dur... mais bon, déjà un dossier éliminé, je démarre plus vite que la semaine dernière! (http:// www.forumfr.com/sujet892-post50751-le-bar-forumfr.html, $10 / 8 / 2015)$

Enfin, dans (7) et (8), l'opérateur apparaît à l'intérieur du segment de discours correspondant au point de vue B (respectivement, $\mathrm{Sa}$ passagère n'était que légèrement blessée et Le trafic était dense sur la petite ceinture et sur les principales voies d'accès à la capitale):

7) Le pilote était en arrêt cardiaque à l'arrivée des secours. Sa passagère n'était en revanche que légèrement blessée. (http:// www.estrepublicain.fr/edition-haut-doubs/2015/07/18/ morteau-depassement-mortel-pour-un-motard, 9/8/2015)

8) Les conditions de circulation dans le centre de Bruxelles étaient assez bonnes, lundi matin, premier jour du nouveau plan de circulation dans le Pentagone. Le trafic était par contre dense sur la petite ceinture et sur les principales voies d'accès à la capitale. (http://actualite-generale.dhnet.be/_belgique/ville-debruxelles-yvan-mayeur-flou.html, 31/7/2015)

Pour rendre compte de ce que la combinaison de l'opérateur en revanche avec le segment de discours correspondant au point de vue B apporte au sens des extraits (3), (5) et (7), on avancera - en première approximation - que $B$ se trouve mis en contraste avec ce qui précède pour en restreindre la portée, pour le "nuancer», la représentation discursive répondant au schéma "en revanche $\mathrm{B}$ " constituant une "compensation", une contrepartie "positive pour le locuteur» (Hamma \& Haillet, 2002: 110; Haillet, 2007: 61).

Un raisonnement analogue à propos des extraits (4), (6) et (8) conduit à dire que la combinaison de l'opérateur par contre avec le segment de discours correspondant au point de vue $\mathrm{B}$ y joue un rôle 
similaire - à un détail près: la représentation discursive répondant au schéma "par contre $\mathrm{B}$ » ne saurait être qualifiée, dans ces exemples, de «contrepartie positive» ni de «compensation», la «nuance» qu'elle apporte mettant clairement un bémol à ce qui précède.

S'il semble donc possible, à ce stade de l'analyse, de retenir la notion de "contrepartie discursive» (cf. Hamma \& Haillet, 2002: 108) pour caractériser la nature des représentations discursives «en revanche $\mathrm{B}$ » et "par contre $\mathrm{B}$ », de nombreux exemples attestés montrent clairement que la combinaison «en revanche $\mathrm{B}$ » ne représente pas nécessairement B comme «contrepartie positive pour le locuteur»:

9) En bref, Service compris! est un petit jeu amusant, convivial, très sympa et avec de chouettes illustrations, qui permettra de se divertir en famille ou entre amis. En revanche, c'est dommage que les joueurs éliminés n'aient d'autres choix que de s'embêter jusqu'à la fin de la partie. (http://avenjoueurs.com/servicecompris-bromleydion/, 29/8/2015)

10) Les raviolis sont très étonnants avec une sauce à l'intérieur légèrement sucrée. Sans doute les meilleurs que j’aie mangés jusqu'ici. Le porc est décevant en revanche: pas très goûtu. (http:// www.yelp.fr/biz/autour-du-yangtse-paris-2, 10/08/2015)

11) L'hôtel bénéficie d'un emplacement privilégié «les pieds dans l'eau» puisque la plage est située dans le prolongement du jardin. Le cadre est en revanche décevant. La plage se résume, en effet, à une mince bande de sable gris parsemée de galets et bordée de hauts talus. (http://www.easyvoyage.com/hotels/estepona/lasdunas-beach-hotel-spa-1218, 23/8/2015)

Ce phénomène est, en outre, indépendant de la position de l'opérateur par rapport au segment de discours correspondant au point de vue $B$.

De même, la combinaison "par contre $\mathrm{B}$ » - là encore, indépendamment de la position de l'opérateur par rapport au segment de discours correspondant au point de vue $\mathrm{B}$ - peut parfaitement représenter $\mathrm{B}$ comme "positif pour le locuteur»:

12) Iniesta, son talent était tellement évident que je n'ai pas vraiment de mérite. Par contre, je suis content d'avoir dit il y a cinq ans que Piqué deviendrait un jour un titulaire indiscutable de la sélection et du Barça. (http://www.sofoot.com/le-barca-n-aforme-personne-123026.html, 11.8.2015)

13) Les produits sont bons, certes, mais aucune fantaisie, aucune surprise, de la cuisine réalisable à la maison! Et le dessert très 
écoeurant à base de chocolat n'a pas pu être fini. Le service était parfait par contre et le pianiste nous a enchantés. (http://www. lafourchette.com/restaurant/lasserre/250?page $=7,10 / 8 / 2015$ )

14) Le 18 mai nous quittons Uyuni avec beaucoup de nostalgie. Nous savons que nous ne reviendrons pas de sitôt vu l'isolement de cette région! Nous sommes par contre très heureux de quitter ce froid glacial et d'entamer notre descente vers la plaine amazonienne qui s'étale en creux au milieu du continent sud américain. (http://runamsud.blogspot.fr/2014/06/histoires-dun-paysplurinational.html, 9/8/2015)

En effet, comme signalé dans Hamma \& Haillet (2002: 109-112), les opérateurs par contre et en revanche sont, pour certains locuteurs, considérés comme interchangeables (en revanche jouant alors le rôle de "variante soutenue» de par contre) - alors que d'autres locuteurs n'emploient spontanément en revanche qu'en combinaison avec un segment de discours correspondant à un point de vue "positif». Ce phénomène de variation linguistique au sein de la communauté francophone reflète la tension entre deux attitudes: d'un côté, la réticence à employer par contre - condamné par certains puristes ${ }^{5}$ - et son remplacement systématique par en revanche (du moins à l'écrit); de l'autre, l'usage qui réserve en revanche à l'expression d'un «équilibre heureusement rétabli» (Grevisse, 1964: 994, \$980) et qui consiste à ne voir dans par contre que la marque d'une relation d'opposition, ce qui permet à cet opérateur de se combiner tant avec un «avantage» qu'avec un «inconvénient».

Considérant qu'il est impossible d'aller au-delà de ce constat de coexistence de deux types d'usage (et, partant, de déterminer la propriété fondamentale des combinaisons "en revanche B» et "par contre $\mathrm{B}$ » en termes de points de vue positifs - ou négatifs - pour le locuteur), on focalisera l'attention, dans ce qui suit, sur d'autres caractéristiques des représentations discursives en question.

L'hypothèse présentée dans Hamma \& Haillet (2002: 108) consiste à expliquer le fonctionnement de par contre en recourant à la notion de «contrepartie discursive», le nom «contrepartie» y étant pris au sens de

5 Tradition qui remonte à Voltaire et qui est encore bien présente aujourd'hui, à en croire des débats nourris sur Internet... Cf. aussi Hamma \& Haillet (2002: 109-110); Grevisse \& Goosse (2008: 1199). 
"chose qui s'oppose à une autre en la complétant ou en l'équilibrant», l'adjectif «discursive» servant à préciser que la relation qui s'établit entre B et A est construite par l'énonciation de «A par contre B». Dans ce qui suit, on optera pour une définition légèrement différente du sens que l'on donne à "contrepartie», en adoptant la formule «opinion qui s'oppose à une autre et la complète ${ }^{6} »$.

C'est sur cette formule que l'on s'appuiera ici pour proposer une description de la propriété fondamentale commune aux opérateurs en revanche et par contre.

\section{Pour une définition de l'invariant sémantique des opérateurs en revanche et par contre}

On avancera qu'employer «en revanche $\mathrm{B}$ » ou "par contre $\mathrm{B}$ » revient invariablement à opposer le point de vue explicite $\mathrm{B}$ à un point de vue explicite ou implicite $\mathrm{A}$ et à le représenter comme destiné à compléter le point de vue A. La relation d'opposition construite entre $\mathrm{B}$ et $\mathrm{A}$ pour compléter A correspond, dans l'hypothèse présentée ici, à l'invariant sémantique des opérateurs en revanche et par contre, la «nuance» de «rétablissement de l'équilibre» - ou encore de «compensation"- venant éventuellement s'y ajouter en fonction de l'environnement discursif de l'opérateur employé.

Cette proposition permet notamment de rendre compte des cas de figure illustrés par les exemples (15) et $(16)^{7}$ où l'opérateur introduit le segment de discours correspondant au point de vue explicite B:

15) Par définition, un synode est une assemblée délibérative; il est donc habituel qu'en interne des positions divergentes s'expriment. En revanche, c'est la première fois à ma connaissance que, de manière externe et publique, des cardinaux prennent des positions aussi divergentes et aussi tranchées. (http://www.la-croix.

6 Cf. par exemple René Journet, Jacques Petit, Guy Robert, Mots et dictionnaires, tome II (1798-1878), Paris: Les Belles Lettres, 1968, p. 345, ainsi que CNRTL (http:// www.cnrtl.fr/definition/academie8/contrepartie) qui reprend la définition donnée dans la $8^{e}$ édition (1932-1935) du Dictionnaire de l'Académie Française.

7 C'est tout à fait délibérément que l'on choisit ici des exemples où le cotexte gauche de l'opérateur facilite la «détection» (Nølke, 2006b) du point de vue A - représenté comme devant, selon le locuteur, être complété par la prise en compte du point de vue $B$. 
com/Religion/Actualite/C-est-la-premiere-fois-que-de-tellesdivergences-s-expriment-publiquement-2014-09-18-1208198, 9/8/2015)

16) $A u$ vu du contexte, l'ARS ne table pas sur une réouverture prochaine du lac. "La prolifération des cyanobactéries était un risque identifié: cela s'était déjà produit il y a quelques années. Par contre, c'est la première fois à ma connaissance que cela arrive pendant le mois d'août. » (http://www.larepubliquedespyrenees. $\mathrm{fr} / 2015 / 08 / 17 /$ les-algues-freinent-la-saison-estivale-de-labase-de-loisirs, 1269950.php, 25/8/2015)

Dans ces deux exemples, le point de vue $\mathrm{B}$ correspond à l'intégralité du cotexte droit de l'opérateur employé. La relation d'opposition s'établit avec, respectivement, le caractère habituel des divergences entre cardinaux dans (15) et le caractère prévisible de la prolifération des cyanobactéries dans (16). Dans les deux cas, le point de vue B correspond à ce qu'il y a d'inédit dans l'événement spécifique commenté - sans que l'on puisse parler raisonnablement de "compensation" ou encore de « rétablissement de l'équilibre».

Les exemples (17) et (18) - où l'opérateur figure à l'intérieur du segment de discours correspondant au point de vue explicite B - sont à cet égard similaires à (15) et à (16):

17) Il était long de 3,5 mètres, pesait environ 400 kilos et ressemblait à un lion. Il possédait les canines les plus longues $(23 \mathrm{~cm})$ et l'apophyse la plus proéminente de tous les nimravidés. Le volume de sa boîte crânienne était en revanche très petit par rapport à d'autres félins. (https://fr.wikipedia.org/wiki/Barbourofelis, 11/8/2015)

18) De façon à s'assurer un début d'exercice sans déficit hydrique, l'American College of Sports Medicine (1996) recommande de boire de 400 à $600 \mathrm{ml}$ d'eau, deux heures avant le début de l'exercice. Ce laps de temps devrait permettre à l'athlète d'uriner les excédents de liquide. Ces recommandations sont, par contre, très générales et les besoins de l'athlète pourraient être plus importants, particulièrement par température chaude et humide et lorsqu'une grande quantité de sueur est déversée durant les deux heures précédant l'exercice. (Chatard, Lutter contre le dopage en gérant la récupération physique, Publications de l'Université de Saint-Étienne, 2005)

Dans ces deux extraits, si le point de vue B s'oppose bien à ce qui est dit dans son cotexte gauche, il ne constitue pas pour autant une 
«compensation» par rapport au point de vue A - qu'il ne fait que compléter.

Il en va de même dans (19) et (20), où l'opérateur figure immédiatement à droite du segment de discours correspondant au point de vue B:

19) Cette femme huissier était venue [...] remettre une convocation pour une saisie. Le pli concernait une personne absente à l'heure du dépôt de l'avis. Le frère du destinataire était là en revanche. Et le ton est vite monté entre l'huissier et le locataire qui a fini par faire manger la convocation à celle qui était venue l'apporter. Un geste qui lui vaudra une comparution en justice le 21 novembre. (http://www.sudouest.fr/2013/07/18/faits-divers-1118244-4584. php, 10/8/2015)

20) Au Portugal, on raffole des lupins, à l'apéritif. Juste cuits à l'eau avec $d u$ sel. La farine, je ne connaissais pas, par contre. (http:// www.cleacuisine.fr/gateaux/clea-a-teste-pour-vous-la-farinede-lupin, 7/8/2015)

Dans (19), c'est à l'absence du destinataire de la convocation que s'oppose la présence de son frère; le point de vue $B$ complète le point de vue A (et constitue l'étape nécessaire pour assurer la cohérence de la suite de la petite histoire...) sans que l'on puisse envisager de parler de «compensation» ou de «rétablissement de l'équilibre». De même, dans (20), qui est un commentaire (reproduit ici dans son intégralité) publié sur un site présentant une recette de gâteau à base de farine de lupin, le point de vue $\mathrm{B}$ «La farine, je ne connaissais pas» - paraphrasable par "Je ne connaissais pas la farine de lupin"-s'oppose au point de vue A paraphrasable par «Je connais bien les graines de lupin ${ }^{8}$ » et ne fait que le compléter, sans pour autant constituer une «compensation» ni «rétablir l'équilibre».

Bien évidemment, le point de vue B peut être représenté non seulement comme une "contrepartie discursive» du point de vue $\mathrm{A}$ auquel il s'oppose et qu'il complète, mais aussi comme constituant une «compensation» et/ ou comme "rétablissant l'équilibre»:

21) Le Nissan Note est doté d'un design intérieur très sobre en comparaison à ses concurrents. En revanche, il est confortable

8 L'exemple (20) est à cet égard similaire à l'exemple (2) cité supra (... ça, en revanche, je connais.). 
et astucieux. Banquette rabattable, coffre à double plancher et beaucoup de luminosité compensent largement ce choix d'une esthétique simple. (http://www.sixt.fr/location-nissan/note/, 21/8/2015)

22) Le pare-brise est ajustable, mais il faut s'arrêter et utiliser les deux mains pour faire varier sa position. Par contre, il est efficace, il protège bien des intempéries, et il engendre peu de turbulences et peu de bruit de vent. (http://www.motojournalweb.com/ articles/30295/, 11/8/2015)

Dans (21), le point de vue B complète le point de vue A en opposant certains avantages du véhicule en question à la simplicité de son design intérieur, ces avantages étant représentés comme compensant largement ce qui aurait pu être considéré comme un handicap par rapport aux modèles concurrents. C'est une dynamique discursive similaire qu'illustre (22), en opposant le point de vue B portant sur l'efficacité (et d'autres qualités) du pare-brise de moto décrit par cet extrait à ce qui pourrait être considéré comme des "imperfections" et qui correspond au point de vue A. C'est dans ce type d'exemples que le fonctionnement des opérateurs en revanche et par contre est similaire à celui de l'opérateur mais évoqué au $\$ 1$ : le discours représente son locuteur comme prédisposé à admettre - et comme cherchant à faire admettre - les conclusions déductibles du point de vue B (et non celles que favorise le point de vue A).

Cela étant, l'observation et l'analyse des exemples (15) à (20) plaident en faveur de l'hypothèse défendue ici et qui consiste à considérer que la propriété fondamentale des représentations «en revanche $\mathrm{B}$ » et "par contre $\mathrm{B}$ »-celle qui se manifeste dans tous leurs emplois - est d'opposer le point de vue explicite B à un point de vue explicite ou implicite A et de le représenter comme destiné à compléter le point de vue A, le discours ainsi construit n'étant pas nécessairement orienté vers les conclusions déductibles du point de vue $\mathrm{B}$.

C'est bien ce que l'on observe notamment dans (23) et (24), exemples qui illustrent une fonction discursive tout à fait courante (cf. Hamma \& Haillet, 2002: 108) des représentations «en revanche $\mathrm{B}$ » et «par contre $\mathrm{B} »$ :

23) Notre confrère Julien Martin ne compte plus les «Julien Martin» qui se bousculent dans la sphère numérique. [...] Mais, hormis quelques tracas administratifs, il s'accommode assez bien de 
cette foule: "C'est comme être anonyme. C'est pratique, les gens croient que c'est un pseudo." Le nom de Mohamed Merah n'était en revanche pas suffisamment fréquent pour préserver la tranquillité de Mohamed Merah, boxeur à Douai. Et surtout, avant la tuerie de Toulouse, en 2012, le sportif avait le malheur d'être plus présent sur le web que son homonyme. Dès que le nom du tueur au scooter a filtré, des médias peu précautionneux ont donc vite affirmé, "googling» à l'appui, que le boxeur et lui ne faisaient qu'un. (L'Observateur $\mathrm{n}^{\circ} 2608,2014$ : 87)

L'extrait (23), qui a pour objet l'homonymie et ses conséquences à l'ère du numérique, fait apparaître la diversité des cas observés sans en représenter aucun comme prédominant ou "privilégié »; l'emploi de l'opérateur en revanche sert à opposer deux exemples A et B et à compléter le premier par le second sans que les conclusions déductibles de B l'emportent sur celles que favorise A.

De même, dans l'extrait (24), la mise en opposition des points de vue $A$ et $B$ vient illustrer l'assertion qui sert d'ouverture:

24) Cet inventaire des diverses possibilités explique que l'on trouve tant de différences entre les peuples: ainsi, en Polynésie, il n'existe pas en général de rites de puberté, l'enfant devient progressivement adolescent, ce n'est que par le mariage qu'il passe à l'état adulte et ce sont ici les cérémonies du mariage qui sont prépondérantes; par contre, en Mélanésie, c'est le passage de l'enfance à l'adolescence qui est abrupt, et les rites de mariage n'ont plus la même importance. ${ }^{9}$

Là encore, "par contre $\mathrm{B}$ " représente le point de vue $\mathrm{B}$ comme s'opposant au point de vue A et comme destiné à le compléter pour appuyer ce qui est dit à propos des différences entre les peuples sur le plan des rites de puberté et de mariage; l'orientation de l'extrait (24) est déterminée non pas par des conclusions déductibles du point de vue $\mathrm{B}$ à lui seul, mais par la mise en relation du point de vue $\mathrm{B}$ avec le point de vue $A$.

9 Exemple emprunté à Masseron \& Wiederspiel (2001/2003: 332), et commenté dans Hamma \& Haillet (2002: 108). Masseron \& Wiederspiel (2001/2003: 331-334) montrent notamment que dans ce type de constructions, l'emploi de "par contre B " représente $\mathrm{B}$ comme un contre-exemple s'opposant à la généralisation à partir de $\mathrm{A}$ formule qui permet aussi d'expliquer le fonctionnement de «en revanche $\mathrm{B}$ » dans l'exemple (23). 


\section{Conclusion}

Fondée sur l'observation et l'analyse d'une variété d'exemples attestés, la démarche visant à rendre compte du fonctionnement discursif des opérateurs en revanche et par contre - en vue de proposer une définition de l'invariant sémantique commun à tous leurs emplois - conduit à postuler que les représentations désignées par les schémas «en revanche $\mathrm{B}$ » et «par contre $\mathrm{B}$ » mettent un point de vue explicite $B$ en relation avec un point de vue explicite ou implicite A et qu'ils représentent invariablement $\mathrm{B}$ à la fois comme s'opposant à $\mathrm{A}$ et comme destiné à le compléter. La relation ainsi construite par le discours a pour effet de représenter le point de vue B comme une «contrepartie discursive» du point de vue A.

Une telle définition de la nature des représentations «en revanche $\mathrm{B}$ » et "par contre $\mathrm{B}$ » revient à considérer qu'elles ont une fonction discursive fondamentale, celle qui se manifeste "toujours et partout», et qu'à cette fonction fondamentale peuvent «localement» s'ajouter d'autres fonctions: en tant que «contrepartie discursive» de A, B peut éventuellement - mais non nécessairement - se trouver interprété comme «compensant» A ou encore comme «rétablissant l'équilibre» par rapport à $A$.

L'approche présentée ici consiste à raisonner en termes de points de vue et s'inscrit résolument dans une conception polyphonique du discours (appréhendé en tant qu'ensemble plus ou moins complexe de représentations de «ce dont on parle»), l'analyse sémantique d'un opérateur discursif ayant systématiquement pour objectif de cerner la nature de la relation qu'il établit entre points de vue.

\section{Bibliographie}

ANSCOMBRE J.-C. \& DUCROT O. (1976), L'argumentation dans la langue, Langages 42, 5-27.

ANSCOMBRE J.-C. \& DUCROT O. (1977), Deux mais en français?, Lingua 43, 23-40.

ANSCOMBRE J.-C. \& DUCROT O. (1981), Interrogation et argumentation, Langue française 52, 5-22.

ANSCOMBRE J.-C. \& DUCROT O. (1983), L'argumentation dans la langue, Bruxelles, Mardaga. 
BRUXELLES S. et al. (1976), Mais occupe-toi d'Amélie, Actes de la recherche en sciences sociales 6, 47-62.

DUCROT O. (1980), Analyse de textes et linguistique de l'énonciation, in Ducrot O. et al., Les mots du discours, Paris, Minuit, 7-56.

DUCROT O. (1984), Le dire et le dit, Paris, Minuit.

GREVISSE, M. (1964), Le bon usage, Gembloux, Duculot.

GREVISSE, M. \& GOOSSE, A. (2008), Le bon usage (14e édition), Bruxelles, De Boeck et Larcier.

HAILLET P.P. (2004), Cadres hypothétiques au conditionnel et stratégie concessive, in Donaire M.-L. (éd.), Dynamiques concessives, Madrid, Arrecife, 95-116.

HAILlET P.P. (2007), Pour une linguistique des représentations discursives, Bruxelles, De Boeck.

HAILLET P.P. (2010), Du moins et au moins: fondements d'une approche contrastive, Revue de Sémantique et Pragmatique 28, 47-72.

HAILLET P.P. (2014), La modalisation et la médiativité en tant que stratégies discursives, in Anscombre J.-C., OppermannMarsauxE., Rodríguez Somolinos A. (éds), Médiativité, polyphonie et modalité en français, Paris, Presses de la Sorbonne Nouvelle, 51-66.

HAMMA B. \& HAILLET P.P. (2002), Par contre: un type particulier de dynamique discursive, LINX 46, 103-113.

MASSERON C. \& WIEDERSPIEL B. (2001/2003), Contrastivité adverbiale: AU CONTRAIRE, CONTRAIREMENT À, PAR CONTRE, in Perroz P. (éd.), Contre: identité sémantique et variation catégorielle, Université de Metz, coll. Recherches linguistiques 26, 311-341.

NØLKE H. (2002), Donc, revenons à nos moutons! Contraintes grammaticales sur le repérage des arguments des connecteurs, in Jansen H. et al. (eds), L'infinito e oltre. Omaggio a Gunver Skytte, Odense, Odense University Press, 373-390.

NØLKE H. (2006a), Connecteurs pragmatiques. Apport de quelques connecteurs à la structure polyphonique, Le français moderne $74: 1,32-42$.

NØLKE H. (2006b), Connexion et anaphoricité: une étude du connecteur par contre, in Riegel M., Schnedecker C., Swiggers P., Tamba I. (éds), Aux carrefours du sens. Hommages offerts à Georges Kleiber pour son $60^{e}$ anniversaire, Louvain, Peeters, 423-439.

PRIETO L. (1966), Messages et signaux, Paris, P.U.F. 